Federal Reserve Bank of Minneapolis

Research Department

\title{
Calibration and Bayesian Learning
}

\author{
Nurlan Turdaliev* \\ Working Paper 596
}

September 1999

\begin{abstract}
In a repeated game of incomplete information, myopic players form beliefs on next-period play and choose strategies to maximize next-period payoffs. Beliefs are treated as forecast of future plays. Forecast accuracy is assessed using calibration tests, which measure asymptotic accuracy of beliefs against some realizations. Beliefs are calibrated if they pass all calibration tests. For a positive Lebesgue measure of payoff vectors, beliefs are not calibrated. But, if payoff vector and calibration test are drawn from a suitable product measure, beliefs pass the calibration test almost surely.
\end{abstract}

JEL classification numbers: C10, C70, C72

*Turdaliev, Federal Reserve Bank of Minneapolis and University of Minnesota. The support of the NSF is gratefully acknowledged. I am grateful to Professor James Jordan for bringing my attention to the problem, his advice, and encouragement. I am grateful to Professor Edward Green for many conversations and very helpful suggestions. I also thank participants of the Spring 1999 Midwest Economic Theory Meeting and the Tenth International Conference on Game Theory at SUNY Stony Brook for helpful comments. The views expressed herein are those of the author and not necessarily those of the Federal Reserve Bank of Minneapolis or the Federal Reserve System. 


\section{Introduction}

Nash equilibrium as a solution concept has been a center of attention in economics for half a century. Yet the problem of explaining how players come to play it has no satisfactory solution. Bayesian learning literature (e.g., Jordan 1991, Jordan 1997, Kalai and Lehrer 1993, Nyarko 1994) is one attempt to address this issue. In case when players' individual characteristics (e.g., payoff matrices) are private information, several authors including the ones mentioned above have recently shown that infinitely repeated play of the same game will result in convergence of players' beliefs to Nash equilibrium of the one-shot game. In a one-shot game Nash equilibrium players' beliefs coincide with the strategies played. If a one-shot game has a unique pure strategy Nash equilibrium, then in the repeated game beliefs and actual plays would converge to each other (both will converge to the Nash equilibrium). But when the one-shot game has a mixed strategy Nash equilibrium, beliefs and actual plays need not converge to each other. The relevance of beliefs in predicting actual plays becomes a nontrivial issue. This paper studies Bayesian learning processes treating beliefs as forecast of actual plays, and assesses the accuracy of this forecast using the notion of calibration.

Bayesian learning in games can be modeled in several alternative ways. One possible approach (we follow it in this paper) is to assume "bounded rationality" of the players and Bayesian update of their beliefs. Each player has an initial belief (or forecast) about his opponents' strategies in the first period; his first period strategy is a best response to this forecast. If a player's strategy is a mixed one, he randomizes over his actions. Then a play, i.e. action profile, is realized. As time passes, the player updates his belief/forecast using the Bayes rule, and chooses best response to the forecast as his strategy for the next period, etc.

The notion of calibration (see Dawid 1982, Foster and Vohra 1997, Kalai et al. 1995, Lehrer 1997) can be used to evaluate the quality of a forecast. This is one of several criteria that have been used to test the reliability of probability forecasts. Let us comment on the result by Kalai et al. (1995) that makes this criterion particularly attractive. A forecast is said to merge to the true distribution if, as time passes, forecasted probabilities converge to the true probabilities. Intuitively, merging seems to be an appropriate criterion to measure the accuracy of the forecast. So does calibration, as Kalai et al. (1995) prove the equivalence between calibration and merging. In this paper we show that in a repeated game of incomplete information about the payoff functions, if Nature is to choose a vector of players' payoff functions according to Lebesgue measure, then with positive probability it will choose such payoffs that players' forecasts are not calibrated with the "true" distribution of plays. Or, using the equivalence result by Kalai et al. (1995), the players' forecasts and the actual plays do not converge to each other. In other words, the players' predictions in those games do not become asymptotically accurate. The def-

inition of calibration requires that forecast pass all calibration tests, basic ingredients of calibration. The set of calibration tests is large (of cardinality continuum). We relax the 
requirements in the definition of calibration to address this question: To what extent are the players' forecasts accurate? Our main result states that if Nature arbitrarily chooses a game (players' payoff matrices) according to Lebesgue measure, as well as a calibration test according to some probability distribution, then with probability one players' forecasts will pass the calibration test.

Let us give some more details. The environment is the same as that in Jordan (1991) and Jordan (1997). We consider finite normal form games of incomplete information played repeatedly infinitely many times. The number of payers and number of actions available to each player are finite and fixed. Then a game is specified by choosing payoff matrix for each player. There is a large set of possible games (of positive Lebesgue measure). Each player knows his payoff matrix but not those of the other players. Players are assumed to have a common prior distribution over the set of possible games. Nature first chooses a game according to that common prior distribution. Every player observes only his component of the game, i.e. his payoff matrix. Suppose that in period 1 players choose their strategies according to a Bayesian Nash equilibrium. This means that every player forms a belief about other players' period 1 strategies. After every finite history of plays, each player can observe all the previously chosen actions. He then updates (according to the Bayes rule) his prior distribution over the set of possible games, and then chooses a new strategy for next period according to a new Bayesian Nash equilibrium. In doing so the player has a certain belief about what the other players will play next period. These formation of beliefs (if some other conditions are met) is called sophisticated Bayesian learning process (SBLP). ${ }^{1}$ Beliefs of the players (they are compatible in SBLP) form a belief about what will be played by all players next period. One can interpret this belief as a forecast. Then the question arises as to how good this forecast might be. Obviously, it would depend on the true game that Nature has chosen.

In the following two paragraphs we will give an intuitive definition of calibration using a weather forecast example. ${ }^{2}$ Suppose the state of weather can be either $R$ (rain) or $S$ (shine) in any period; there are infinite number of periods $t=1,2, \ldots$; there is a true distribution $\mu$ of infinite histories (sequences) of $R$ and $S$. A forecast $\nu$ should assign a probability distribution over set $\{R, S\}$ after every finite history of $R$ and $S$. One possible way of assessing the forecast $\nu$ is to compare, after any finite sequence of realizations of $R$ and $S$, the empirical frequency of, say, $R$ over the sequence with average forecast of $R$ over the same sequence. Intuitively, if forecast is "good" then these two numbers should be close to each other as time passes. The idea behind calibration is to compare these two variables calculated not only over the entire sequences, but also over all subsequences. Kalai et al. (1995) motivate this approach by the following example. Suppose the true distribution $\mu$ puts probability one on sequence $(R, S, R, S, \ldots)$, and

\footnotetext{
${ }^{1}$ One can argue that if Bayesian Nash equilibrium is involved in the definition of this learning process, then it cannot be a foundation of Nash equilibrium. There is an alternative non-Bayesian way of defining SBLP. In formal part of this paper we follow this approach. For details, see Jordan (1997).

${ }^{2}$ The formal definition can be found in section 2 .
} 
forecast $\nu$ assigns distribution $(0.5,0.5)$ after any finite sequence. Intuitively, it is not a good forecast since it cannot detect a very simple pattern $(R, S, R, S, \ldots)$. If we compare empirical frequencies of $R$ with average predictions of $R$ over periods $t=1,2, \ldots, T$ along $(R, S, R, S, \ldots)$, those numbers will be close to each other, suggesting that the forecast is "good". But calibration allows to calculate those variables over any increasing sequence of periods. Let us compute the two variables over odd periods $t=1,3,5, \ldots, T$ (assuming odd $T)$. Empirical frequency of $R$ along $(R, S, R, S, \ldots)$ is 1 , whereas average prediction of $R$ over the same history is still 0.5 . The two numbers cannot become close to each other no matter how large $T$ is. Thus, calibration can detect the "badness" of $\nu$. This was possible because we could specify when to check the forecast: only in odd periods along $(R, S, R, S, \ldots)$.

Roughly, a calibration test, which is a basic ingredient of calibration, specifies when to check the forecast (in the example above it is all histories of odd length along $(R, S, R, S, \ldots)$, and what event to check (in the example the event is $\{R\}$ ). A forecast $\nu$ passes a calibration test with respect to the true distribution $\mu$ if along $\mu$-almost every infinite history empirical frequencies converge to the average predicted probabilities, where both are calculated according to the calibration test (as in the example). Forecast $\nu$ is calibrated with respect to the true distribution $\mu$ if it passes every calibration test with respect to $\mu$.

Let us make clear how we use the parallel between "forecast vs. true distribution" and "beliefs vs. actual plays". Consider a SBLP, denote it $e$. As we argued above, it can be viewed as a forecast of what will be played next period. Suppose Nature has chosen a game $g$ according to the common prior. (As we said earlier, each player now can see his own component, or payoff matrix, of the game, but not those of other players.) This game induces a new probability measure $e_{g}$ of histories that will actually be played. We can treat this induced distribution as the true distribution for this game. In this paper we concern ourselves with the question of whether for every (or almost every) game $g$ (chosen first by Nature) the SBLP $e$, as a forecast, is calibrated with the induced probability distribution $e_{g}$ of actually played histories.

If one wants to show that beliefs in a Bayesian learning process, treated as forecast, are "good" in some sense, a desirable result would be to show that for all/almost all games the forecast is calibrated, or, alternatively, a $\operatorname{SBLP} e$ is calibrated with all/almost all $e_{g}$. We prove that this statement is not true. It is shown that for a set of games of positive Lebesgue measure the forecast is not calibrated. On the other hand, we show that under certain conditions for almost every game the forecast is "almost" calibrated.

More specifically, a calibration test tells for every finite history of plays whether to check the players' expectations about what will be played next period, and if yes, specifies what event to check. A SBLP deterministically passes a calibration test at a game if observed long-run empirical frequencies of plays equal the average forecasted probabilities along all best-response histories of the game (an infinite history of plays $\left(h_{1}, h_{2}, \ldots\right)$ is a best response of a game if period $t$ play $h_{t}$ is a myopic best response to expectations conditioned on the observed history $\left.\left(h_{1}, \ldots, h_{t-1}\right)\right)$. A SBLP is strictly calibrated at a 
game if it deterministically passes all calibration tests at that game. We show that for a fixed SBLP there is a positive measure of games at which the SBLP is not strictly calibrated.

This result shows that with positive probability Nature can choose such a game that players' beliefs do not converge to the actual plays, i.e. their forecasts are not asymptotically accurate. There is a connection between this result and those of Nachbar (1994) and Foster and Young (1996). In Nachbar (1994) it is shown that in a two-player discounted repeated game there is no belief for either player such that two assumptions termed Conventional Prediction and Conventional Optimization hold. Loosely put, these two assumptions require that the players are good predictors of future plays and are rational. The author assumes no uncertainty about the payoffs. Foster and Young (1996), on the other hand, assume both uncertainty about payoffs and myopic maximization as in the present paper. They show that for a large set of games, if players are good predictors and rational, then neither beliefs nor actions converge to a Nash equilibrium.

If, as we have shown, in many games players' forecasts are not asymptotically accurate, can one measure the extent to which these forecasts are accurate? Suppose an inspector checks the accuracy of the forecast. But instead of applying all calibration tests she applies only a subset of calibration tests. The simplest case is when this subset is a singleton. The main result of the paper is that for any fixed calibration test, if Nature chooses a game, with probability one it will be such a game that players' forecasts will pass the calibration test. We generalize this result in the following way. Suppose the inspector chooses calibration test according to some probability distribution. If Nature arbitrarily chooses a game, with probability one it will be such a game that the players' forecasts will pass calibration test with probability one. In other words, the SBLP is "almost" calibrated with respect to almost every game.

Section 2 contains definitions and the results by other authors that we use in the proofs. Section 3 contains our main results. Section 4 concludes.

\section{Preliminaries}

The situation to be formalized here is the following. There is a finite number of players, each has a finite fixed number of actions. Then any game is specified by a collection of payoff matrices of players. There is imperfect information about payoff functions: there is a large set of possible games (vectors of payoff functions); Nature chooses a game according to some probability measure and reveals to each player only his own payoff matrix. Players then play the game repeatedly infinitely many times. In any period, if a player's strategy is mixed, he randomizes. Then Nature chooses a play, i.e. a vector of actions, according to the strategies. All previous plays are observable. After observing the previous period play, every player uses Bayes rule to update his belief about other players' payoff functions, and then forms a belief about next period strategies of other 
players. This formation of beliefs is a learning process.

We will deal with n-player normal form games with $n \geq 2$. For each player $i$, let the set of strategies $S_{i}$ be finite. Let $S=\prod_{i=1}^{n} S_{i}$. As usual, $S_{-i}=\prod_{i \neq j} S_{j}$. The payoff function of player $i$ is $u_{i}: S \rightarrow \Re$, where $\Re$ denotes the set of real numbers. Let the set of possible payoff functions for player $i$ be the unit ball $U=\left\{u \in \Re^{S} \mid\left(\sum_{s \in S} u(s)^{2}\right)^{1 / 2} \leq 1\right\}$. Then the set of all possible games is $G=U^{n}$. For every $g \in G$ let $N(g)$ denote the set of Nash equilibria of $g$. If $A$ is a finite set, $\Delta(A)$ denotes the set of probability distributions on $A$.

Definition 2.1 For each $t \geq 1$, let $H^{t}=\prod_{\tau=1}^{t} S$, with generic element $h^{t}=\left(h_{1}, \ldots, h_{t}\right)$, where each $h_{\tau}=\left(h_{i \tau}\right)_{i=1}^{n}$. Denote $H^{0}=\{*\}$ (initial history). Let $H^{\infty}=\prod_{\tau=1}^{\infty} S$, with generic element $h=\left(h_{1}, h_{2}, \ldots\right)$. For a given $h \in H^{\infty}$, let $h^{t}$ denote -period truncation of $h$. We call any $h$ and $h^{t}$ an infinite and a finite history respectively.

Let $X$ be a topological space. $\mathcal{B}_{X}$ denotes the Borel $\sigma$-algebra on $X$. All measurable spaces in this paper are of type $\left(X, \mathcal{B}_{X}\right)$. Whenever we say "measurable set" we mean "Borel set". If $\left(X, \mathcal{B}_{X}, \mu\right)$ and $\left(Y, \mathcal{B}_{Y}, \nu\right)$ are two measure spaces, we sometimes consider product space $\left(X \times Y, \mathcal{B}_{X} \times \mathcal{B}_{Y}, \mu \times \nu\right)$, where $\mathcal{B}_{X} \times \mathcal{B}_{Y}$ is the product $\sigma$-algebra, $\mu \times \nu$ is the product measure.

If $\mu$ and $\nu$ are measures on a measurable space $\left(X, \mathcal{B}_{X}\right)$, we use notation $\mu \ll \nu$ to indicate that $\mu$ is absolutely continuous with respect to $\nu$.

The following definitions are due to Jordan (1991) and Jordan (1997). ${ }^{3}$

Definition 2.2 A learning process is an n-tuple $e=\left(e_{i}\right)_{i=1}^{n}$, where each $e_{i}=\left\{e_{i t}\right\}_{t=0}^{\infty}$ with $e_{i t}: H^{t} \rightarrow \Delta\left(S_{-i}\right)$.

One can give the following interpretation to a learning process. After any finite history $h^{t}$ (including the initial history) every player $i$ has expectation (or, belief) $e_{i t}\left(h^{t}\right)$ as to what the other players will play next period $t+1$.

Definition 2.3 Let e be a learning process and $g=\left(u_{i}\right)_{i=1}^{n} \in G$. A best-response history for $(e, g)$ is an infinite history $h=\left(h_{1}, h_{2}, \ldots\right) \in H^{\infty}$ such that for each $t \geq 0$ and $i$, $h_{i, t+1}$ maximizes $\sum_{s_{-i} \in S_{-i}} u_{i}\left(\cdot, s_{-i}\right) e_{i t}\left(h^{t}\right)\left(s_{-i}\right)$ over $S_{i} . B R H(e, g)$ will denote the set of best-response histories for $(e, g) .{ }^{4}$ If $B R H(e, g)$ is a singleton, we denote it $h(e, g)$.

In other words, along $h \in B R H(e, g)$ every player myopically maximizes next period expected utility given his expectation about what the other players will play.

In what follows, if $\phi$ is a measure on $G \times H^{\infty}$, the marginals of $\phi$ on $G$ and $H^{\infty}$ will be denoted by $\phi_{G}$ and $\phi_{H}$, respectively. If $\chi$ is a measure on $H^{\infty}$, the marginal of $\chi$ on $H^{t}$ will also be denoted as $\chi$ (this should not cause confusion). Also, if $h^{t+1}=\left(h^{t}, s\right)$ with $s \in S$, we will frequently use $\chi\left(s \mid h^{t}\right)$ instead of $\chi\left(h^{t+1} \mid h^{t}\right)$. If $\chi$ is a Borel measure on a topological space $X$, supp $\chi$ is defined as the smallest closed subset $A$ with $\chi(A)=1$.

\footnotetext{
${ }^{3}$ We have adapted some of them to our case.

${ }^{4}$ Obviously, $B R H(e, g)$ is a nonempty set.
} 
Definition 2.4 Let $\phi$ be a Borel probability measure on $G \times H^{\infty}$ satisfying:

(i) $\phi_{G}=\phi_{G}^{1} \times \cdots \times \phi_{G}^{n}$, where each $\phi_{G}^{i}$ is a probability measure on $U$.

(ii) For each $h^{t} \in \operatorname{supp} \phi, \phi_{H}\left(\cdot \mid h^{t}\right)$ is a product distribution, i.e. $\phi_{H}\left(\cdot \mid h^{t}\right)=$ $\phi_{H}^{1}\left(\cdot \mid h^{t}\right) \times \cdots \times \phi_{H}^{n}\left(\cdot \mid h^{t}\right)$, where each $\phi_{H}^{i}\left(\cdot \mid h^{t}\right)$ is a measure on $S_{i}$ and $\phi_{H}\left(\cdot \mid h^{t}\right)$ is a measure on $S$.

(iii) For each $t \geq 0$, define $e_{t}: H^{t} \rightarrow \Delta(S)$ by ${ }^{5}$

$$
e_{t}\left(h^{t}\right)(s)=\phi_{H}\left(h_{t+1}=s \mid h^{t}\right) .
$$

For each $i$ and $h^{t} \in H^{t}$, let $e_{i t}\left(h^{t}\right)$ be the marginal of $e_{t}\left(h^{t}\right)$ on $S_{-i}$. This defines the learning process e as follows: $e_{i t}: H^{t} \rightarrow \Delta\left(S_{-i}\right)$ and $e_{i}=\left\{e_{i t}\right\}_{t=0}^{\infty}$.

(iv) If $(g, h) \in \operatorname{supp} \phi$, then $h \in B R H(e, g)$.

Then $e=\left(e_{i}\right)_{i}$ is called a sophisticated Bayesian learning process (SBLP) induced by $\phi$.

In Jordan (1991), the distribution $\phi$ is called a Bayesian strategy process (BSP). Jordan (1997) considers a stricter version of SBLP. In particular, besides the conditions in Definition 2.4 it is required that each $\phi_{G}^{i}$ is a u.a.c. probability measure on $U .{ }^{6}$ Jordan (1991) shows (Proposition 2.6) that if $\mu$ is a product measure on $G$, i.e. $\mu=\mu_{1} \times \cdots \mu_{n}$, where each $\mu_{i}$ is an arbitrary measure on $U$, then there exists a SBLP $e$ induced by $\phi$ such that $\phi$ agrees with $\mu$ on $G$. For the case when the "product measure" assumption (ii) in the previous definition is not made general existence conditions are given in Cotter (1991) and Yannelis and Rustichini (1991).

Now we turn to defining the notion of calibration that will be used to assess the quality of players' beliefs treated as forecast. If $A$ is a set, let $\mathcal{P}(A)$ denote the set of subsets of A. The following definition is due Kalai et al. (1995) (from now on KLS).

Definition 2.5 A checking rule is a pair of functions $(C, D)$ such that

$$
\begin{aligned}
& C: \bigcup_{t=0}^{\infty} H^{t} \rightarrow\{0,1\}, \\
& D: \bigcup_{t=0}^{\infty} H^{t} \rightarrow \mathcal{P}(S) .
\end{aligned}
$$

\footnotetext{
${ }^{5}$ If $h^{t} \notin \operatorname{supp}\left(\phi_{H}\right)$, then $e_{t}\left(h^{t}\right)$ is defined arbitrarily.

${ }^{6}$ Let $m$ be Lebesgue measure on $U$. A Borel probability measure $\mu$ on $U$ is uniformly absolutely continuous (u.a.c.) if there are numbers $\alpha>0$ and $\beta>0$ such that $\alpha m(A) \leq \mu(A) \leq \beta m(A)$ for every Borel set $A \subset U$.
} 
The function $C$ indicates whether to check (a forecast against the actual realization) after any finite history, and the function $D$ specifies which event to check. Here we follow Dawid (1982) in restricting ourselves to checking rules that consider only next period events. One could consider checking rules with $D$ being uniformly bounded finite horizon events. KLS argue that this is equivalent to one-period-ahead case (as far as calibration is concerned; see Definitions 2.6 and 2.7 below). Everything is different if there is no upper bound on the horizon of events in $D$ (see KLS).

Note that the set of cluster points of any sequence in $\Delta(S)$ is a nonempty subset of $\Delta(S)$. We say that the sequence $\left\{\sigma_{n}\right\}$ in $\Delta(S)$ converges to the set $A \subset \Delta(S)$ and denote it $\sigma_{n} \rightarrow A$ if all cluster points of $\left\{\sigma_{n}\right\}$ belong to $A$.

We introduce the following two definitions.

Definition 2.6 Let e be a SBLP, $g \in G$, and $(C, D)$ a checking rule. We say that $e$ deterministically passes the calibration test induced by $(C, D)$ at game $g$ (or, shortly, e g-passes the calibration test $(C, D))$, if for every $h \in B R H(e, g)$ satisfying $\sum_{\tau=0}^{\infty} C\left(h^{\tau}\right)=$ $\infty$, the following holds:

$$
\lim _{t \rightarrow \infty} \frac{\sum_{\tau=0}^{t} C\left(h^{\tau}\right)\left[I\left(h^{\tau+1} \in D\left(h^{\tau}\right)\right)-e_{\tau}\left(h^{\tau}\right)\left(D\left(h^{\tau}\right)\right)\right]}{\sum_{\tau=0}^{t} C\left(h^{\tau}\right)}=0,
$$

where $I$ is indicator function.

If the beliefs $e_{\tau}\left(h^{\tau}\right)\left(D\left(h^{\tau}\right)\right)$ are interpreted as predicted probabilities of the events $D\left(h^{\tau}\right)$, then the SBLP $e$ in the previous definition $g$-passes the calibration test $(C, D)$ if along any $h \in B R H(e, g)$ the observed empirical frequencies converge to the average of predictions, where both frequencies and averages are calculated with respect to $C$, i.e. things are ignored when $C\left(h^{\tau}\right)=0$. From now on, we use the terms "checking rule" and "calibration test" interchangeably.

Definition 2.7 We say that a $S B L P$ e is strictly calibrated at a game $g \in G$ (or, shortly, g-calibrated), if e g-passes all calibration tests.

There is a very close relation between the notion of $g$-calibration and the notion of calibration in the Dawid-KLS sense. A forecasting is a function from $\cup_{t=0}^{\infty} H^{t}$ to $\Delta(S)$. Equation (1) shows that a SBLP $e$ can be interpreted as a forecasting. If $\mu$ is a Borel probability measure on $H^{\infty}$, a forecasting $e$ is said to pass a calibration test $(C, D)$ with respect to $\mu$ (in the KLS sense) if there is a set $H^{\prime} \subset H$ with $\mu\left(H^{\prime}\right)=1$ such that every $h \in H^{\prime}$ with $\sum_{\tau=0}^{\infty} C\left(h^{\tau}\right)=\infty$ satisfies (2). A forecasting $e$ is said to be calibrated with $\mu$ (in the KLS sense) if $e$ passes all calibration tests with respect to $\mu$. It turns out (see Theorem 1 below) that for a given $\operatorname{SBLP} e$, the set $B R H(e, g)$ is a singleton for almost every game $g$, so that $B R H(e, g)=\{h(e, g)\}$. Take any such a game $g$. If $\delta_{h(e, g)}$ denotes Dirac measure that puts probability one on point $h(e, g)$, then $e$ is $g$-calibrated if and only if $e$ is calibrated (in KLS sense) with $\delta_{h(e, g)}$. 
As we will see later (see Proposition 1 below), $g$-calibration may be too demanding in our setup because all calibration tests have to be passed. In Section 2 we introduce another version of calibration with a milder requirement that almost all calibration tests be passed.

We will need the following results.

Lemma 1 (Jordan 1997, p.155) The set of games with a mixed Nash equilibrium as the unique Nash equilibrium has positive Lebesgue measure.

We can take a "matching pennies" type of game for $n$ players with two strategies for each player. This game would have a unique Nash equilibrium, and in addition this equilibrium would be a mixed equilibrium. Moreover, all games in an $\epsilon$-neighborhood of $g$ would have the same property. To obtain an $n$-player game with arbitrary number of strategies in sets $S_{i}$ we add strictly dominated strategies. The resulting game would still have the property of the original game as well as all games in its small neighborhood.

Theorem 1 (Jordan 1997, Theorem 1) For every learning process e there is a set of games $G_{e}^{B R H}$ such that Lebesgue measure $m\left(G \backslash G_{e}^{B R H}\right)=0$, and for every $g \in G_{e}^{B R H}$ the set $B R H(e, g)$ is a singleton, i.e. $B R H(e, g)=\{h(e, g)\}$.

Theorem 2 (Jordan 1991, Theorem 3.2) Let e be a SBLP induced by $\phi$. Then

$$
\phi\left(\left\{(g, h) \mid e_{t}\left(h^{t}\right) \rightarrow N(g)\right\}\right)=1 .
$$

The theorem says that for $\phi$-almost every pair (game, infinite history) beliefs along the history converge to the set of Nash equilibria of the game.

Definition 2.8 For each $t \geq 1$, define the empirical frequency distribution $f_{t}: H^{t} \rightarrow \Delta(S)$ by

$$
f_{t}\left(h^{t}\right)(s)=\frac{1}{t} \cdot \#\left\{\tau \leq t \mid h_{\tau}=s\right\}, \quad \text { all } s \in S .
$$

Theorem 3 (Jordan 1997, Theorem 3) ${ }^{7}$ Let e be a SBLP induced by $\phi$. Let $\phi_{G}=$ $\phi_{G}^{1} \times \cdots \times \phi_{G}^{n}$, where each $\phi_{G}^{i}$ is a probability measure on $U$ that is mutually absolutely continuous with respect to Lebesgue measure. Let $G_{e}^{B R H}$ be as in Theorem 2. Then there is a set $G_{e}^{F C} \subset G_{e}^{B R H}$ such that Lebesgue measure $m\left(G \backslash G_{e}^{F C}\right)=0$ and for every $g \in G_{e}^{F C}$, if $h=h(e, g)$ is the unique element of BRH(e,g), then

$$
\lim _{t \rightarrow \infty}\left\|f_{t}\left(h^{t}\right)-\frac{1}{t} \sum_{\tau=1}^{t} e_{\tau-1}\left(h^{\tau-1}\right)\right\|=0 .
$$

\footnotetext{
${ }^{7}$ In Jordan (1997) this theorem is proven with the stronger assumption that each $\phi_{G}^{i}$ is a u.a.c. probability measure on $U$ (see footnote 6), for the author needs that assumption in another theorem (of general convergence). It is easily seen that if just Theorem 3 needs to be proven, the weaker assumption $m \ll \phi_{G}^{i} \ll m$ is enough. See also the discussion after Definition 2.4.
} 
Thus, along the unique best-response history the empirical frequencies of strategy profiles approach their average expected probabilities.

Finally, let us introduce some convenient notation. ${ }^{8} \mathcal{C}$ will denote the set of calibration tests with generic element $c=(C, D)$. For a fixed SBLP $e$ define functions $\zeta_{e}^{t}: \mathcal{C} \times H^{\infty} \rightarrow \Re, t=0,1,2, \ldots$, by ${ }^{9}$

$$
\zeta_{t}^{e}(c, h)=\frac{\sum_{\tau=0}^{t} C\left(h^{\tau}\right)\left[I\left(h^{\tau+1} \in D\left(h^{\tau}\right)\right)-e_{\tau}\left(h^{\tau}\right)\left(D\left(h^{\tau}\right)\right)\right]}{\sum_{\tau=0}^{t} C\left(h^{\tau}\right)},
$$

where $0 / 0=0$. Note that $(2)$ in this notation can be rewritten as

$$
\lim _{t \rightarrow \infty} \zeta_{t}^{e}(c, h)=0
$$

\section{Results}

We first show that there is no hope for a SBLP to be $g$-calibrated at all/almost all games.

Proposition 1 Let e be a SBLP induced by $\phi$. Suppose that the condition of Theorem 3 is satisfied. Then there is a set of games $G_{e}^{N C} \subset G$ with positive Lebesgue measure such that for no game $g \in G_{e}^{N C}$ the $S B L P$ e is g-calibrated.

Proof. Let $G^{0}$ and $G_{e}^{F C}$ be the sets of games described in Lemma 1 and Theorem 3 respectively, and let $G_{e}^{\prime}=G^{0} \cap G_{e}^{F C}$. Then $m\left(G_{e}^{\prime}\right)>0$. Let $T_{e}=\left\{(g, h) \mid e_{t}\left(h^{t}\right) \rightarrow N(g)\right\}$. From Theorem $2 \phi\left(T_{e}\right)=1$. Denote the projection of $T_{e}$ on $G$ by $G_{e}^{\prime \prime}$. Then $\phi_{G}\left(G_{e}^{\prime \prime}\right)=1$ as $\phi\left(T_{e}\right)=1$. Since $\phi_{G}\left(G \backslash G_{e}^{\prime \prime}\right)=0$ and $m \ll \phi_{G}, m\left(G \backslash G_{e}^{\prime \prime}\right)=0$. Let $G_{e}^{N C}=G_{e}^{\prime} \cap G_{e}^{\prime \prime}$. From above $m\left(G_{e}^{N C}\right)>0$, and for every $g \in G_{e}^{N C}$ we have the following:

- the set $N(g)$ is a singleton: $N(g)=\{\sigma(g)\}$, where $\sigma(g)$ is a mixed strategy Nash equilibrium;

- the set $B R H(e, g)$ is a singleton: $B R H(e, g)=\{h(e, g)\}$;

- $e_{t}\left(h^{t}(e, g)\right) \rightarrow \sigma(g)$ as $t \rightarrow \infty$.

This and Theorem 3 imply that

$$
\lim _{t \rightarrow \infty}\left\|f_{t}\left(h^{t}(e, g)\right)-\sigma(g)\right\|=0, \quad \text { all } g \in G_{e}^{N C} .
$$

Fix an arbitrary $g \in G_{e}^{N C}$. Since $\sigma(g)$ is mixed, there are two different $s^{\prime}, s^{\prime \prime} \in S$ with

$$
\sigma(g)\left(s^{\prime}\right)>0, \quad \sigma(g)\left(s^{\prime \prime}\right)>0 .
$$

\footnotetext{
${ }^{8}$ Throughout we assume that $n, S_{1}, \ldots, S_{n}$, and thus $S, U, G, H^{\infty}$, are fixed.

${ }^{9}$ Here and below subscripts and superscripts $e, c$, or $g$ indicate dependence on SBLP $e$, calibration test $c$, or game $g$, respectively.
} 
From (5) and (6) we have that $h_{t}(e, g)=s^{\prime}$ for infinitely many $t$. Define checking rule $c^{e, g}=\left(C^{e, g}, D^{e, g}\right)$ as follows:

for every $h^{t} \in H^{t}$

$$
\begin{aligned}
& C^{e, g}\left(h^{t}\right)= \begin{cases}1 & \text { if } h^{t+1}(e, g)=\left(h^{t}, s^{\prime}\right), \\
0 & \text { otherwise; }\end{cases} \\
& D^{e, g}\left(h^{t}\right)= \begin{cases}\left\{h^{t+1}(e, g)\right\} & \text { if } C^{e, g}\left(h^{t}\right)=1, \\
\emptyset & \text { otherwise. }\end{cases}
\end{aligned}
$$

Then

$$
\begin{aligned}
& I\left[h^{t+1}(e, g) \in D^{e, g}\left(h^{t}(e, g)\right)\right]=1, \quad \text { all } t=0,1,2, \ldots, \\
& e_{t}\left(h^{t}(e, g)\right)\left(D^{e, g}\left(h^{t}(e, g)\right)\right) \rightarrow \sigma\left(s^{\prime}\right) \quad \text { as } t \rightarrow \infty .
\end{aligned}
$$

From above $\sum_{\tau=0}^{\infty} C^{e, g}\left(h^{\tau}(e, g)\right)=\infty$. Therefore ${ }^{10}$

$$
\zeta_{t}^{e}\left[c^{e, g}, h(e, g)\right] \rightarrow 1-\sigma\left(s^{\prime}\right)>0 .
$$

Thus, $g$ does not pass calibration test $c^{e, g}$ and therefore $e$ is not $g$-calibrated.

Proposition 2 Let e be a $S B L P$ induced by $\phi$. Let $m$ be Lebesgue measure on $G$ and $m \ll$ $\phi_{G}$. Then for every calibration test $c$ there is a set of games $G_{e, c}$ such that $m\left(G \backslash G_{e, c}\right)=0$, and e g-passes c for every game $g \in G_{e, c}$.

Proof. Fix a calibration test $c=(C, D)$. By Dawid's theorem ${ }^{11}$ there is a set of histories $H_{e, c} \subset H^{\infty}$ with measure $\phi_{H}\left(H_{e, c}\right)=1$ such that for any history $h \in H_{e, c}$ with $\sum_{t=0}^{\infty} C\left(h^{t}\right)=\infty$ the following holds:

$$
\lim _{t \rightarrow \infty} \zeta_{t}^{e}(c, h)=0
$$

Define $\hat{G}_{e, c} \subset G$ as

$$
\hat{G}_{e, c}=\left\{g \in G \mid \text { there is } h \in H_{e, c} \text { such that }(g, h) \in \operatorname{supp} \phi\right\} .
$$

Since $\phi_{H}\left(H_{e, c}\right)=1$, we conclude that $\phi_{G}\left(\hat{G}_{e, c}\right)=1$.

Define $G_{e, c}=\hat{G}_{e, c} \cap G_{e}^{B R H}$, where $G_{e}^{B R H}$ is from Theorem 1. Note that $\phi_{G}\left(G \backslash \hat{G}_{e, c}\right)=0$ and $m\left(G \backslash G_{e}^{B R H}\right)=0$. Since $m \ll \phi_{G}$, we conclude that $m\left(G \backslash G_{e, c}\right)=0$. From the definition of $G_{e, c}$ we infer that for every game $g \in G_{e, c}$ the set $B R H(e, g)$ is a singleton. From this, (7), and part (iv) of Definition 2.4 we conclude that $e g$-passes $c$ for every $g \in G_{e, c}$.

\footnotetext{
${ }^{10}$ See (3) and (4).

${ }^{11}$ Appendix contains the statement of the theorem and its proof.
} 
The set of calibration tests $\mathcal{C}$ can be naturally topologized (with every basic open set being a set of calibration tests that agree on a finite history $\left.h^{t}\right)$. Let $\mathcal{C}$ be endowed with the $\sigma$-algebra $\mathcal{B}_{\mathcal{C}}$ generated by open sets, i.e. the Borel $\sigma$-algebra.

We will need the following notation. Let $\left(X, \mathcal{B}_{X}, \mu\right)$ and $\left(Y, \mathcal{B}_{Y}, \nu\right)$ be finite measure spaces. If $A \subset X \times Y, x \in X, y \in Y$, the $x$-section $A_{x}$ is defined by $A_{x}=\{y \in Y \mid(x, y) \in$ $A\}$, and similarly, $y$-section $A_{y}=\{x \in X \mid(x, y) \in A\}$. We will use the mathematical fact that if $A \in \mathcal{B}_{X} \times \mathcal{B}_{Y}$, then all $A_{x} \in \mathcal{B}_{Y}, A_{y} \in \mathcal{B}_{X}$ and

$$
\mu \times \nu(A)=\int_{X} \nu\left(A_{x}\right) d \mu(x)=\int_{Y} \nu\left(A_{y}\right) d \nu(y)
$$

(see, e.g., Proposition 2.34 and Theorem 2.36, pp. 63-64 in Folland (1984)).

In spite of the fact that, according to Proposition 2, each $c$-section of the set $E=$ $\{(c, g) \mid e$ does not $g$-pass $c\}$ is a subset of Lebesgue measure zero, in general $E$ might not be measurable and may not be a subset of any null set (see Exercise 2.5.47, p.67 in Folland (1984)). The following lemma and proposition show that, in fact, $E$ is a measurable null set.

Lemma 2 Set $E=\{(c, g) \mid$ e does not g-pass $c\}$ is measurable.

The proof can be found in Appendix.

As before, $m$ will denote Lebesgue measure on the set of games $G$.

Proposition 3 Let e be a SBLP induced by $\phi$. Let $\psi$ be a Borel probability measure on $\mathcal{C}$. Suppose $\phi_{G} \ll m \ll \phi_{G}$. Denote $\chi=\phi_{G} \times \psi$. Then for $\chi$-almost every pair $(c, g) \in \mathcal{C} \times G$ the SBLP e g-passes calibration test c.

Proof. Let $E=\{(c, g) \mid e$ does not $g$-pass $c\}$. By Lemma $2 E$ is measurable. Since both $\phi_{G}$ and $\psi$ are finite measures, we can apply (8):

$$
\chi(E)=\int_{\mathcal{C}} \phi_{G}\left(E_{c}\right) d \psi(c),
$$

where $E_{c}=\{g \in G \mid(c, g) \in E\}$. By Proposition 2 and assumption $\phi_{G} \ll m, \phi_{G}\left(E_{c}\right)=0$ for every $c \in \mathcal{C}$. Thus, $\chi(E)=0$.

Definition 3.1 Let e be a SBLP induced by $\phi, \psi$ a Borel probability measure on $\mathcal{C}$. Let $g \in G$. The $S B L P$ e is said to be $\psi$-calibrated at $g$ if e $g$-passes $\psi$-almost every calibration test.

Corollary 1 Let e be a SBLP induced by $\phi$. Let $\psi$ be a Borel probability measure on $\mathcal{C}$. Suppose $\phi_{G} \ll m \ll \phi_{G}$. Then there exists a set of games $G_{e}^{A C}$ such that $m\left(G \backslash G_{e}^{A C}\right)=0$, and $e$ is $\psi$-calibrated with respect to every $g \in G_{e}^{A C}$.

Proof. Let $E$ be from the proof of Proposition 3, so that $\phi_{G} \times \psi(E)=0$. The claim follows from (8) and the assumption $m \ll \phi_{G}$. 


\section{Conclusions}

Another way to see the intuition behind Proposition 1 is to use the result by Kalai et al. (1995) on the relationship between calibration and merging. Let $\mu$ (true distribution) be a probability measure on $H^{\infty}$ and $\nu$ (forecasting) a function from $\cup_{t=0}^{\infty} H^{t}$ to $\Delta(S)$. Forecasting $\nu$ induces a unique (subjective) probability distribution on $H^{\infty}$ which we (with a slight abuse of notation) also denote $\nu$. Then the subjective distribution $\nu$ is said to merge to $\mu$, if for $\mu$-almost every $h \in H^{\infty}$

$$
\lim _{t \rightarrow \infty} \max _{A \subset S}\left|\mu\left(A \mid h^{t}\right)-\nu\left(A \mid h^{t}\right)\right|=0 .
$$

The main result in KLS is that calibration and merging are equivalent, i.e. $\nu$ is calibrated with $\mu$ if and only if $\nu$ merges to $\mu$. As we said earlier ${ }^{12}$, for almost every game $g$ a SBLP $e$ is calibrated at $g$ if and only if $e$ is calibrated with $\delta_{h(e, g)}$. From KLS's equivalence result it follows that if $e$ were calibrated at almost every game $g$, then expectations along the unique $\mathrm{BRH} h(e, g)$ would have merged to $\delta_{h(e, g)}$ as $(9)$ suggests. But since there is a set of games with positive Lebesgue measure that have mixed equilibrium as a unique Nash equilibrium, and since expectations along (unique) BRH must converge to Nash equilibrium by Jordan (1997), this is impossible. In other words, the main culprit of Proposition 1 as a negative result is the fact that for many games (of positive Lebesgue measure) players cannot "learn" to play mixed Nash equilibrium.

Jackson et al. (1998) are interested in representing a probability distribution governing a stochastic process in the form

$$
\mu=\int_{\Theta} \mu_{\theta} d \lambda(\theta)
$$

so that each component $\mu_{\theta}$ is both "learnable" ( $\mu$ merges to $\mu_{\theta}$ ) and "sufficient for prediction" (knowing $\mu_{\theta}$ is enough to predict in the sense that the past realizations would not help in prediction). This representation reflects a two stage Bayesian process in which Nature first chooses $\mu_{\theta}$ according to measure $\lambda$, and then $\mu_{\theta}$ governs the evolution of the process. Jackson et al. (1998) argue that if one does not impose the two requirements on the components $\mu_{\theta}$, then there are infinitely many representations (10). But if one does impose them, then for a large class of processes such a representation exists and is unique. In our case we represent a SBLP $e$ as

$$
e=\int_{G} \delta_{h(e, g)} d \phi_{G}
$$

Proposition 1 shows that components $\delta_{h(e, g)}$ are not "learnable" for a large set of games $g$ (though it is easily seen that they are "sufficient to predict"). In the language of Jackson et al. (1998), this representation is "too fine" to be learnable.

\footnotetext{
${ }^{12}$ See the discussion after Definition 2.7.
} 
Proposition 2 shows that if an inspector uses just a single calibration test, then a SBLP passes that test at almost all games. The key argument in the proof of Proposition 2 is that we fix calibration test $c$ and then find set $G_{e, c}$. If the set of calibration tests were countable then by straightforward argument we would have shown that for almost all games $g$, a SBLP $e$ is calibrated at $g$. But the set of calibration tests has cardinality continuum. Nevertheless, we can generalize Proposition 2 in the following way. Suppose ${ }^{13}$ the inspector chooses calibration tests according to a probability distribution $\psi$ over the set of calibration tests. If Nature simultaneously chooses a game according to Lebesgue measure, then with probability one it will be such a game that SBLP passes $\psi$-almost all calibration tests (or, SBLP is $\psi$-calibrated).

\section{Appendix A}

Proof of Lemma 2 Observe that

(i) for any $t=0,1,2, \ldots$, the set $\left\{\zeta_{t}^{e}(c, h) \mid(c, h) \in \mathcal{C} \times H^{\infty}\right\}$ is a finite subset of $\Re$;

(ii) for any pair $(\hat{c}, \hat{h}) \in \mathcal{C} \times H^{\infty}$ and any $t=0,1,2, \ldots$, the set of pairs $(c, h)$ agreeing with $(\hat{c}, \hat{h})$ on $\hat{h}^{t}$ is an open set, thus a Borel set.

These two observations imply that all $\zeta_{t}^{e}$ are measurable functions. Therefore (see, e.g., Problem 3, p. 47 in Folland (1984)) the set

$$
A^{\prime \prime}=\left\{(c, h) \mid \lim _{t \rightarrow \infty} \zeta_{t}^{e}(c, h) \text { exists }\right\}
$$

is measurable, as well as the set

$$
A^{\prime \prime \prime}=\left\{(c, h) \mid \lim _{t \rightarrow \infty} \zeta_{t}^{e}(c, h)=0\right\}
$$

We will show that the set

$$
A^{\prime}=\left\{(c, h) \mid \sum_{t=0}^{\infty} C\left(h^{t}\right)=\infty\right\}
$$

is measurable. Since the sets

$$
A_{t, T}^{\prime}=\left\{(c, h) \mid \sum_{\tau=0}^{t} C\left(h^{\tau}\right) \geq T\right\}, \quad \text { all } t=0,1,2, \ldots, \quad 0 \leq T \leq t+1,
$$

\footnotetext{
${ }^{13}$ Lehrer (1997) first used the approach of passing almost all calibration tests as opposed to passing all calibration tests in a different context. The main result of his paper is that for a given probability measure $\psi$ over $\mathcal{C}$, the forecaster can find a forecast that would pass $\psi$-almost all calibration tests, or in other words, any inspection is manipulable.
} 
are measurable, and

$$
A^{\prime}=\bigcap_{T=1}^{\infty} \bigcup_{t=T-1}^{\infty} A_{t, T}^{\prime}
$$

$A^{\prime}$ is measurable.

Define

$$
A=\left(\left(\mathcal{C} \times H^{\infty}\right) \backslash A^{\prime \prime}\right) \bigcup\left(A^{\prime} \cap\left(A^{\prime \prime} \backslash A^{\prime \prime \prime}\right)\right)
$$

It is easy to see that $A \subset A^{\prime}$. In words, $A$ is a set of pairs $(c, h)$ such that $\sum_{t=0}^{\infty} C\left(h^{t}\right)=\infty$ and either $\lim _{t \rightarrow \infty} \zeta_{t}^{e}(c, h)$ does not exist, or $\lim _{t \rightarrow \infty} \zeta_{t}^{e}(c, h) \neq 0$. $A$ is measurable, of course.

Let us show that the set

$$
B=\{(h, g) \mid h \in B R H(e, g)\}
$$

is measurable. Indeed, sets

$$
\begin{aligned}
& B\left(\hat{h}^{t}\right)=\left\{(h, g) \mid h^{t}=\hat{h}^{t} \text { and there exists } \bar{h} \in B R H(e, g) \text { with } \bar{h}^{t}=\hat{h}^{t}\right\}, \\
& \text { all } t=0,1,2, \ldots, \hat{h}^{t} \in H^{t},
\end{aligned}
$$

are measurable. For

$$
B=\bigcap_{t=0}^{\infty} \bigcup_{\hat{h}^{t} \in H^{t}} B\left(\hat{h}^{t}\right)
$$

$B$ is measurable.

Consider the measurable set $E^{\prime}=A \times G \cap \mathcal{C} \times B$. From above, $E^{\prime}$ is the set of triples $(c, h, g)$ such that $h \in B R H(e, g), \sum_{t=0}^{\infty} C\left(h^{t}\right)=\infty$, and either $\lim _{t \rightarrow \infty} \zeta_{t}^{e}(c, h) \neq 0$ or this limit does not exists. Then $E$ is the projection of $E^{\prime}$ on $\mathcal{C} \times G$ :

$$
E=\left\{(c, g) \mid \text { there exists } h \in H^{\infty} \text { s.t. }(c, h, g) \in E^{\prime}\right\} .
$$

Therefore $E$ is a measurable set.

\section{Appendix B}

For completeness we include the statement and proof of Dawid's theorem. Recall that $e_{t}\left(h^{t}\right)(\cdot)=\phi_{H}\left(\cdot \mid h^{t}\right)$ (see Definition 2.4). The theorem says that $e$ is calibrated with itself (in KLS sense). See also the discussion after Definition 2.7.

Theorem 4 (Dawid 1982) Let e be a SBLP induced by $\phi$. For every calibration test $c=(C, D)$ there is a set of histories $H_{e, c} \subset H^{\infty}$ with $\phi_{H}\left(H_{e, c}\right)=1$ such that for every $h \in H_{e, c}$ with $\sum_{t=0}^{\infty} C\left(h^{t}\right)=\infty$ the following holds:

$$
\lim _{t \rightarrow \infty} \frac{\sum_{\tau=1}^{t}\left[I\left[h^{\tau} \in D\left(h^{\tau-1}\right)\right]-e_{\tau-1}\left(h^{\tau-1}\right)\left(D\left(h^{\tau-1}\right)\right)\right] C\left(h^{\tau-1}\right)}{\sum_{\tau=1}^{t} C\left(h^{\tau-1}\right)}=0 .
$$


Proof. $\quad$ Define the random variables $\alpha_{t}: H^{\infty} \rightarrow \Re$ and $\beta_{t}: H^{\infty} \rightarrow \Re$ as follows:

$$
\begin{aligned}
\alpha_{t}(h) & =\frac{I\left[h^{t} \in D\left(h^{t-1}\right)\right]-e_{t-1}\left(h^{t-1}\right)\left(D\left(h^{t-1}\right)\right)}{\sum_{\tau=1}^{t} C\left(h^{\tau-1}\right)} C\left(h^{t-1}\right), \\
\beta_{t} & =\sum_{\tau=1}^{t} \alpha_{\tau}, \\
t=1,2, \ldots, &
\end{aligned}
$$

where $0 / 0=0$. It is easy to see that $E\left(\alpha_{t} \mid h^{t-1}\right)=0$ (here expectation is taken with respect to measure $\left.\phi_{H}\right)$. Hence, $\left\{\beta_{t}\right\}$ is a martingale.

Let us check that the condition of the martingale convergence theorem is met (see Feller (1966), Theorem VII.8.1, p.236). It is sufficient to show that the second moment of $\left\{\beta_{t}\right\}$ is uniformly bounded. Indeed, since $\left(I\left[h^{t} \in D\left(h^{t-1}\right)\right]-e_{t-1}\left(h^{t-1}\right)\left(D\left(h^{t-1}\right)\right)\right)^{2} \leq 1$,

$$
E\left(\alpha_{t}^{2}\right) \leq E\left(\left[\frac{C\left(h^{t-1}\right)}{\sum_{\tau=1}^{t} C\left(h^{\tau-1}\right)}\right]^{2}\right)
$$

Now we show that $E\left(\alpha_{t} \cdot \alpha_{t^{\prime}}\right)=0$ if $t<t^{\prime}$. Iterate conditional expectations to obtain $E\left(\alpha_{t} \cdot \alpha_{t^{\prime}}\right)=E\left(\alpha_{t} \cdot E\left(\alpha_{t^{\prime}} \mid h^{t}\right)\right)=0$. Then

$$
\begin{aligned}
E\left(\beta_{t}^{2}\right)=\sum_{\tau=1}^{t} E\left(\alpha_{\tau}^{2}\right) & \leq \sum_{\tau=1}^{t} E\left(\left[\frac{C\left(h^{\tau-1}\right)}{\sum_{s=1}^{\tau} C\left(h^{s-1}\right)}\right]^{2}\right) \\
& =E\left(\sum_{\tau=1}^{t}\left[\frac{C\left(h^{\tau-1}\right)}{\sum_{s=1}^{\tau} C\left(h^{s-1}\right)}\right]^{2}\right) \\
& \leq \sum_{k=1}^{t} \frac{1}{k^{2}}<\sum_{k=1}^{\infty} \frac{1}{k^{2}}=\frac{\pi^{2}}{6}
\end{aligned}
$$

By applying the martingale convergence theorem to $\left\{\beta_{t}\right\}$ we conclude that $\left\{\beta_{t}\right\}$ converges almost surely, i.e. there is a subset $H_{e, c} \subset H^{\infty}$ with $\phi_{H}\left(H_{e, c}\right)=1$ such that $\left\{\beta_{t}(h)\right\}$ converges for all $h \in H_{e, c}$. Then if $h \in H_{e, c}$ and $\sum_{t=0}^{\infty} C\left(h^{t}\right)=\infty$, convergence of

$$
\beta_{t}(h)=\sum_{\tau=1}^{t} \frac{I\left[h^{\tau} \in D\left(h^{\tau-1}\right)\right]-e_{t-1}\left(h^{t-1}\right)\left(D\left(h^{\tau-1}\right)\right)}{\sum_{k=1}^{\tau} C\left(h^{k-1}\right)} C\left(h^{\tau-1}\right)
$$

implies by Kronecker's lemma (see Feller (1966), p.238) that (11) holds. 


\section{References}

Cotter, K. (1991). "Correlated Equilibrium in Games with Type-Dependent Strategies," J. Econ. Theory 54, 48-68.

DAwID, A.P. (1982). "The Well Calibrated Bayesian," J. Amer. Statistical Assoc. 77, 605-613.

FEller, W. (1966). An Introduction to Probability Theory and Its Applications, vol.2. New York: John Wiley \& Sons.

Folland, G. (1984). Real Analysis. Toronto: John Wiley \& Sons.

Foster, D., And Vohra, R. (1997). "Calibrated Learning and Correlated Equilibrium," Games Econ. Behav. 21, 40-55.

Foster, D., And Young, H.P. (1996). "Learning with Hazy Beliefs," Working Paper No. 370, Department of Economics, The Johns Hopkins University.

Jackson, M., Kalai, E., Smorodinsky, R. (1998). "Bayesian Representation of Stochastic Processes under Learning: de Finetti Revisited," Discussion Paper No. 1228, MEDS Department, Northwestern University.

Jordan, J. (1991). "Bayesian Learning in Normal Form Games," Games Econ. Behav. $3,60-81$.

Jordan, J. (1997). "Bayesian Learning in Games: A Non-Bayesian Perspective," in The Dynamics of Norms (C. Bicchieri, R. Jeffrey and B. Skyrms, Eds.), pp. 149-174. Cambridge: Cambridge Univ. Press.

Kalai, E., AND Lehrer, E. (1993). "Rational Learning Leads to Nash Equilibrium," Econometrica 61, 1019-1045.

Kalai, E., Lehrer, E., And Smorodinsky, R. (1995). "Calibrated Forecasting and Merging," Discussion paper No. 1144, MEDS Department, Northwestern University.

LEHrer, E. (1997). "Any Inspection Is Manipulable," mimeo.

NACHBAR, J. (1997). "Prediction, Optimization, and Learning in Repeated Games," Econometrica 65, 275-309.

Nyarko, Y. (1994). "Bayesian Learning Leads to Correlated Equilibria in Normal Form Games," Econ. Theory, 4, 821-841.

Yannelis, N., And Rustichini, A. (1991). "On the Existence of Correlated Equilibria," in Equilibrium Theory with Infinitely Many Commodities (M. Khan and M. Yannelis, Eds.), pp. 268-280. New York/Heidelberg/Berlin: Springer-Verlag. 